\title{
Aurea diagnosis of pneumomediastinum. A case report
}

\author{
Lucio Brugioni, ${ }^{1}$ Francesca De Niederhausern, ${ }^{1}$ Chiara Gozzi ${ }^{1}$, Pietro Martella, ${ }^{1}$ Elisa Romagnoli, ${ }^{1}$ \\ Beatrice Aramini, ${ }^{2}$ Federica Bocchi,${ }^{3}$ Caterina Solieri ${ }^{3}$ \\ ${ }^{1}$ Internal Medicine, Critical Area Unit, Policlinico Modena; ${ }^{2}$ Division of Thoracic Surgery, Policlinico Modena; ${ }^{3}$ Emergency \\ Medicine, University of Modena and Reggio Emilia, Italy
}

\begin{abstract}
Pericarditis and spontaneous pneumomediastinum are among the pathologies that are in differential diagnoses when a patient describes dorsal irradiated chest pain: if the patient is young, male, and long-limbed, it is necessary to exclude an acute aortic syndrome firstly. We present the case of a young man who arrived at the Emergency Department for chest pain: an echocardiogram performed an immediate diagnosis of pericarditis. However, if the patient had performed a chest X-ray, this would have enabled the observation of pneumomediastinum, allowing a correct diagnosis of pneumomediastinum and treatment. The purpose of this report is to highlight the importance of the diagnostic process.
\end{abstract}

\section{Introduction}

Pneumomediastinum (PM) is defined as the presence of air between the structures of the mediastinum. It can be traumatic or spontaneous (SPM) and divided into primary or secondary SPM, depending on whether an underlying pathology that predisposes to the escape of air is present. ${ }^{1}$ SPM was first described in 1939 by Hamman, who observed subcutaneous and mediastinal emphysema in a woman who had just

Correspondence: Lucio Brugioni, Internal Medicine, Critical Area Unit, Policlinico Modena, via del Pozzo 71, 41121 Modena, Italy.

Tel.: +39.059.422549

E-mail: brugioni.lucio@aou.mo.it

Key words: Spontaneous pneumomediastinum; Hamman sign; electrocardiogram; chest X-ray; chest computerized tomography.

Contributions: all authors contributed equally to the writing of the article.

Conflict of interests: the authors declare no potential conflict of interests.

Ethics approval: not required.

Informed consent: the patient gave informed consent.

Received for publication: 16 April 2020.

Revision received: 21 May 2020

Accepted for publication: 15 June 2020

This work is licensed under a Creative Commons Attribution NonCommercial 4.0 License (CC BY-NC 4.0).

${ }^{\circ}$ Copyright: the Author(s), 2020

Licensee PAGEPress, Italy

Italian Journal of Medicine 2020; 14:179-183

doi:10.4081/itjm.2020.1292 given birth; hence, we can refer to the syndrome as Hamman syndrome. ${ }^{2}$

Macklin later described that the pathophysiology of SPM is based on the presence of an alveolarinterstitial gradient: the increase in intra-alveolar pressure leads to the rupture of the walls of the alveoli, generating the propagation of air inside the tissues via dissection, from here, the air diffuses via centripetal forces toward the soft tissues of the neck, the mediastinum, and the pericardium (a phenomenon called the Macklin effect $t^{3,4}$ ).

The primary clinical manifestation is typically retrosternal, pleuritic chest pain radiating to the neck and shoulders. Dyspnea, cough, odynophagia, and/or dysphagia may also be present. ${ }^{5}$ Objectively, in $18 \%$ of cases, synchronous rattles with the heartbeat due to the presence of mediastinal air (Hamman's sign) are observed. ${ }^{2,5}$ Although there are some objective and instrumental aspects that turn out to be diriment, this presentation is also common in patients with acute pericarditis.

In our case, the diagnosis of pneumomediastinum was made later, upon observation of the exploratory computerized tomography angiogram, because of the lack of some crucial radiological and biohumoral steps that would have directed us toward the best therapeutic choices. Thus, for adequate differential diagnosis, it is essential to adhere as much as possible to a method that allows us to organize and guide our diagnostic hypotheses to reach the final diagnosis through an appropriate and minimally invasive path.

\section{Case Reports}

On November 13, 2019, a 19-year-old man arrived at the emergency room due to retrosternal shooting 
respirophasic chest pain that radiated posteriorly to the shoulders and worsened in the supine decubitus and on the left side. He had no respiratory or gastrointestinal flu-like symptoms in the previous days; in particular, he had no fever, cough, or vomiting. Both the remote pathological and the pharmacological anamnesis were silent. He participated in competitive sports (football), and his last stress test performed the previous month was negative.

Vital parameters at the admission were normal: arterial pressure $110 / 70 \mathrm{mmHg}$, cardiac frequency 85 $\mathrm{bpm}$, respiratory frequency 16 acts $/ \mathrm{min}$, oxygen saturation $98 \%$, temperature $36.3^{\circ} \mathrm{C}$, Glasgow coma scale 15 . The general objectivity was almost normal, except for pericardial frictions appreciable on a precordial level; heart tones were rhythmic and tachycardic. Electrocardiogram (Figure 1) showed a sinus rhythm with a frequency average at upper limits, a right focal block, and nonspecific alterations of repolarization. Biohumoral tests showed a mild leukocytosis (white cells $15.20 \mathrm{migl} / \mathrm{mmc}$, reference $<10.90$ ), and abnormalinflammation indexes: $C$ reactive protein (CRP) was $<0.2 \mathrm{mg} / \mathrm{dL}$ and myocardial enzymes absent (cTnI $<12 \mathrm{mg} / \mathrm{L}$ ).

Given the auscultatory finding, a cardiologist evaluated the patient and performed the echocardiogram: the patient was tachycardic, and the acoustic accesses were scarce; despite this, there was minimal pericardial detachment in the inferiorposterior area that was not hemodynamically significant and was associated with hyper-refraction of the pericardial sheets. Diagnosis of acute pericarditis was made, leading to hospitalization under a semiintensive regime for monitoring and therapy [ibuprofen $1800 \mathrm{mg} /$ day divided into three administrations, colchicine $0.5 \mathrm{mg} /$ day (patient was $55 \mathrm{~kg}$ ), and lansoprazole $30 \mathrm{mg}$ ] was started. The next day, the patient was in good hemodynamic compensation, and the pain seemed to be improving, the inflammation markers showed minimal elevation (CRP $1.8 \mathrm{mg} / \mathrm{dL}$ ).

However, the diagnosis of pericarditis was not sufficient to explain some of the data; for example, the absence of symptoms and signs of infection, as well as the electrocardiogram trace that did not show the expected alterations. In addition, the quality of the ultrasound examination was poor and did not allow good visualization of the cardiac morphology and, above all, of the aortic root. Given the acute onset, the extension of pain into the back, the marfanoid habitus, and the D-dimer value not available, the man was urgently subjected to computerized tomography (CT) angiogram study due to the suspicion of an acute aor-
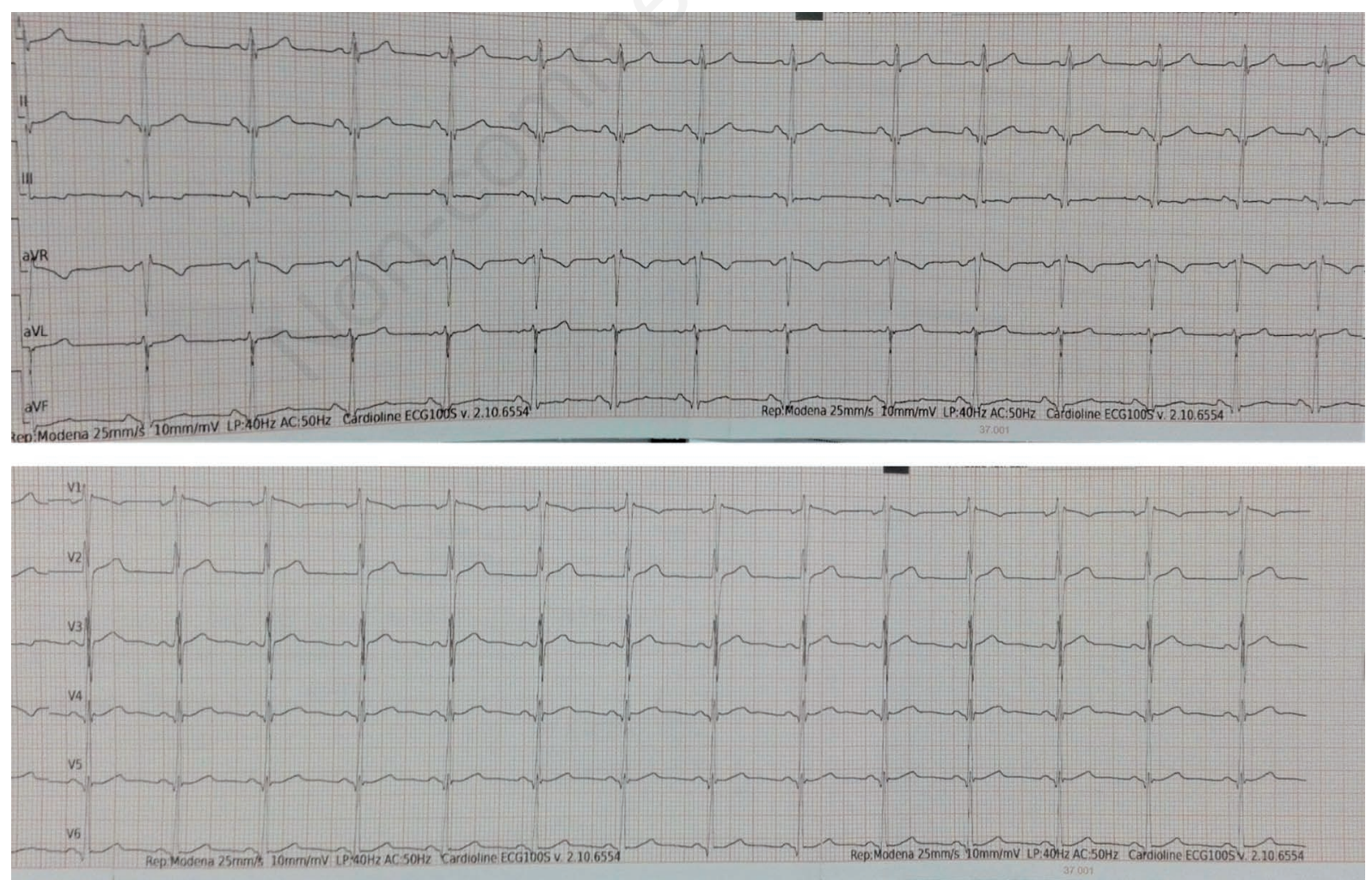

Figure 1. Electrocardiogram, normal sinus rhythm at a rate of 85 beats/minute, aspecific alteration of the repolarization phase, regular PR interval. 
tic syndrome. The investigation showed (Figure 2) no signs of aortic dissection, but it documented the presence of pneumomediastinum extended cranially to the lateral cervical regions, bilaterally associated with a very thin stratum of pneumopericardium, no

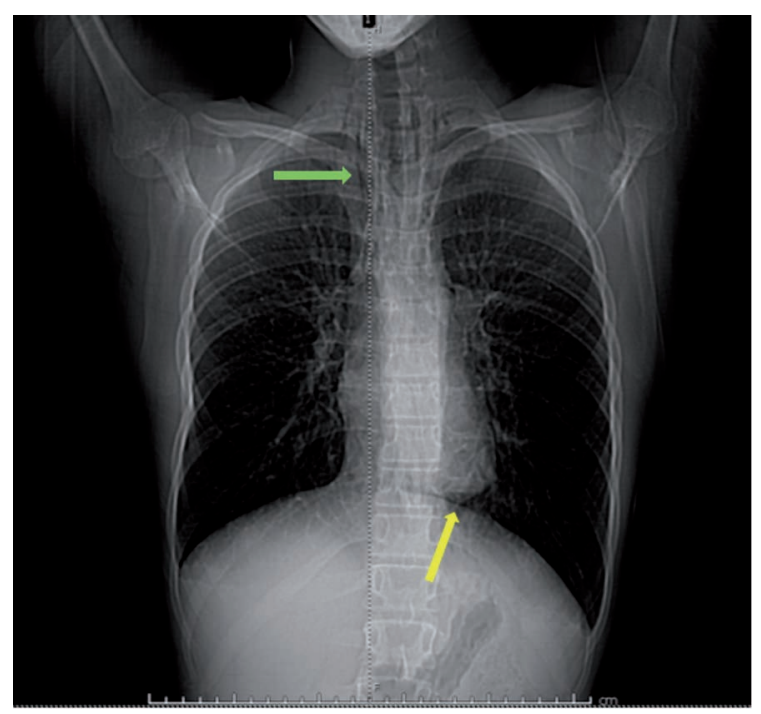

Figure 2. Computed tomographic angiography scout that it is almost a chest radiogram - mediastinal gas is seen as a thin lucent line that seems to separate the heart form the superior surface of the diaphragm (yellow arrow). Linear lucencies overlying the upper chest and neck outlining mediastinal pleura (green arrow). parenchymal lesions or pleuro-pericardial effusion were observed, the trachea was normal, and there were no wall lacerations. Given these data, we completed neck physical examination that was normal, in particular, it showed the absence of subcutaneous emphysema, and a chest X-ray (that had never been done yet) finally was performed that confirmed gas outlining inner surface of mediastinal pleura.

In search of the possible causes of pneumomediastinum, we decided to go ahead with the investigation by performing an esophagography with contrast medium to exclude esophageal lesions. The execution of an additional echocardiogram did not document morphofunctional alterations; therefore, the pericarditis therapy set at the entrance was suspended. The patient was evaluated by a thoracic surgeon that did not identify complications. A control chest X-ray was performed before the discharging from Hospital, and partial reabsorption of the pneumomediastinum layer (4 mm vs $10 \mathrm{~mm}$ ) was noted (Figure 3). On the same day, the inflammation indexes were decreasing (CRP $0.9 \mathrm{mg} / \mathrm{dL}$ ) and the patient was asymptomatic, eupneic in room air, and in proper hemodynamic compensation; thus, he was discharged after 6 days of hospitalization with the recommendation to rest for approximately twenty days and use paracetamol as needed in case of pain. At the radiological check, carried out ten days later, complete reabsorption of the pneumomediastinum layer was observed (Figure 4), and from the clinical point of view, the patient was eupneic and in good respiratory compensation (oxygen saturation 99\%).
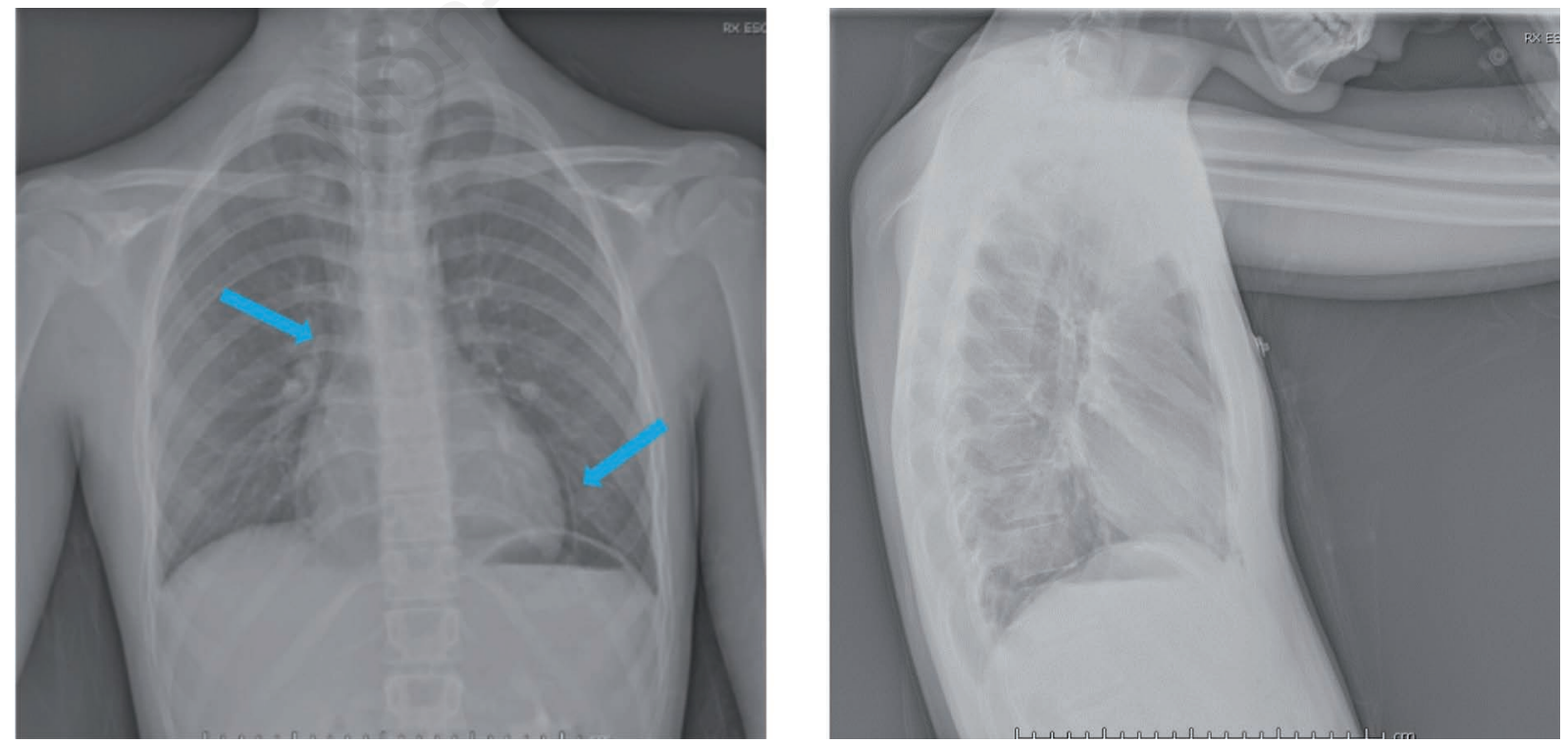

Figure 3. Frontal and lateral chest radiography, November 16. It shows gas outlining the inner surface of mediastinal pleura (blue arrows). 

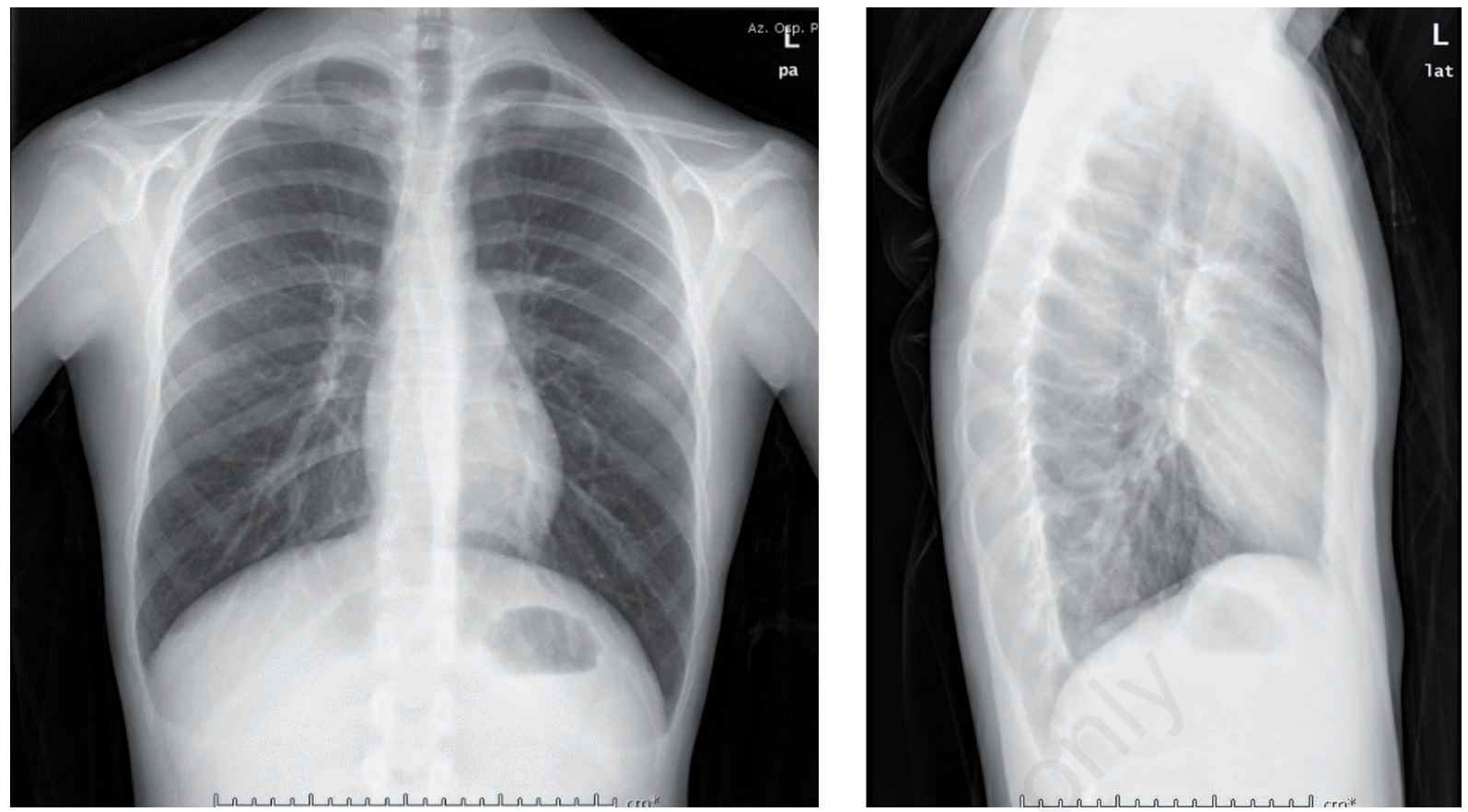

Figure 4. Frontal and lateral chest radiography, November 25. No more pneumomediastinum signs.

\section{Discussion}

Pneumomediastinum is a rare disease, constituting 1 out of 44,500 emergency room visits. A first incidence peak is observed immediately after the neonatal period, the second during early childhood and a third in adolescence. In addition, male subjects, particularly those who are tall and thin, are at higher risk of developing SPM. ${ }^{1,6}$ It is, therefore, more frequent in young subjects since their tissues, that are looser, more elastic, and softer (flaccid), favor the diffusion of gases.

Chest pain is the main symptom of pneumomediastinum (present in $60-100 \%$ of cases): positional, usually retrosternal and radiates to the neck and back, it worsens following deep inhalation. ${ }^{5}$ From an objective point of view, heart tones can be reduced, and it is possible to appreciate near the apex and in the left parasternal seat some rattles synchronous with cardiac systole: this is the Hamman sign, specific to pneumomediastinum, but present in only a small percentage of patients. ${ }^{7}$

These clinical features are shared with acute pericarditis, ${ }^{8-10}$ a pathology to consider for differential diagnosis. It is therefore crucial, although it requires considerable clinical experience, to distinguish pericardial rubbings that have a three-phase trend (that is, audible during atrial systole, ventricular systole, and the rapid filling phase of the early ventricular diastole $^{10}$ ) from the sign of Hamman and pleuritic rubbing. The finding of chest pain and pericardial rubbing, two of the four major diagnostic criteria, would be sufficient to diagnose acute pericarditis, ${ }^{9}$ yet they are two findings superimposable on the clinical presentation of pneumomediastinum.

In our case, echocardiogram was an additional diagnostic support, as it identified the detachment and hyper-echogenicity of the pericardial sheets; however, the acoustic accesses were scarce even though the patient was young and thin (excellent habitus for the propagation of ultrasound).

The air gap represents the ultrasound data observable in pneumomediastinum: this is the presence of airborne artifacts that hinder the vision of the heart coordinated with the respiratory cycle. ${ }^{11}$ Thus, the poor acoustic quality of the image can be justified by the presence of air in the mediastinal compartment.

Comparing the instrumental investigations necessary for the diagnosis of pericarditis ${ }^{9}$ with those of pneumomediastinum, ${ }^{5}$ it emerges that simple chest radiography is the fundamental investigation in the diagnosis of both pathologies and must be carried out first. It can lead to the diagnosis of SPM in $90 \%$ of cases and is also recommended for the diagnosis of acute pericarditis: in the latter case, it could show signs of pleuro-pericardial involvement or, if there was copious pericardial effusion, an increase in the cardiothoracic ratio. ${ }^{9}$

The patient who was the subject of this report underwent a chest X-ray only after hospitalization, 
CT, and diagnosis of SPM. The radiolucent striae characteristic of SPM, ${ }^{7,12}$ which had already emerged in the exploratory CT angiogram, was confirmed later by the chest X-ray, where the cardiac silhouette and the mediastinal pleura stood out due to the air present at the mediastinum level, a sign that is referred to as the continuous diaphragm sign. ${ }^{12}$

\section{Conclusions}

Having neglected the chest X-ray confused our assessments and delayed the correct diagnosis. This case report suggests that, to confirm a diagnostic hypothesis, it is essential not only to find corroborating data but also to exclude those pathologies with typical clinical and laboratory aspects. This case, therefore, underlines how it is essential to use diagnostic tools that allow us to make differential diagnoses. Our diagnostic discernment must be supported by following a method, putting our ideas in order, and, above all, acting proportionately in light of the symptoms and signs: avoiding fixation errors to reach the correct diagnosis.

\section{References}

1. Kouritas VK, Papagiannopoulos K, Lazaridis G, et al. Pneumomediastinum. J Thorac Dis 2015;7:S44-9.

2. Hamman L. Spontaneous mediastinal emphysema. Bull
Johns Hopkins Hosp 1939;64:1-21.

3. Macklin CC. Transport of air along sheaths of pulmonic blood vessels from alveoli to mediastinum: clinical implications. Arch Intern Med 1939;64:913.

4. Wintermark M, Schnyder P. The Macklin effect: A frequent etiology for pneumomediastinum in severe blunt chest trauma. Chest 2001;120:543-7.

5. Chalumeau M, Le Clainche L, Sayeg N, et al. Spontaneous pneumomediastinum in children. Pediatr Pulmonol 2001;31:67-75.

6. Vasileios K. Kouritas, Konstantinos Papagiannopoulos et al. Pneumomediastinum. J Thorac Dis 2015;7:S44-9.

7. Imazio M, Gaita F, LeWinter M. Evaluation and treatment of pericarditis: a systematic review. JAMA 2015;314:1498.

8. Adler Y, Charron P, Imazio M, et al. 2015 ESC Guidelines for the diagnosis and management of pericardial diseases: The Task Force for the Diagnosis and Management of Pericardial Diseases of the European Society of Cardiology (ESC) Endorsed by: The European Association for Cardio-Thoracic Surgery (EACTS). Eur Heart J 2015;36:2921.

9. Spodick DH. Pericardial rub. Prospective, Multiple observer investigation of pericardial friction in 100 patients. Am J Cardiol 1975;35:357.

10. Ng L, Saul T, Lewiss RE. Sonographic evidence of spontaneous pneumomediastinum. Am J Emerg Med 2013;31:462.e3.

11. Zylak CM, Standen JR, Barnes GR, Zylak CJ. Pneumomediastinum revisited. Radiographics 2000;20:1043.

12. Levin B. The continuous diaphragm sign. A newly-recognized sign of pneumomediastinum. Clin Radiol 1973;24:337. 